\title{
CEP Calculation Based on Weighted Bayesian Mixture Model
}

\author{
Shengdi Zhang, ${ }^{1}$ Xiaojun Duan, ${ }^{1}$ Chang Li, ${ }^{1}$ Xiaojun Peng, ${ }^{2}$ and Qiang Zhang ${ }^{2}$ \\ ${ }^{1}$ College of Science, National University of Defense Technology, Changsha, Hunan 410073, China \\ ${ }^{2}$ Rocket Force Equipment Academy, Beijing 100085, China \\ Correspondence should be addressed to Xiaojun Duan; xj_duan@163.com
}

Received 16 June 2016; Revised 29 November 2016; Accepted 26 December 2016; Published 24 January 2017

Academic Editor: Alessandro Tasora

Copyright (C) 2017 Shengdi Zhang et al. This is an open access article distributed under the Creative Commons Attribution License, which permits unrestricted use, distribution, and reproduction in any medium, provided the original work is properly cited.

\begin{abstract}
$\mathrm{CEP}$ is an important accuracy index in the test evaluation for guidance weapon systems. As for the samples origin from diverse populations, which is a general case in the practice, traditional method with one population is inaccurate to give estimate directly. A weighted method considering the credibility of the prior information is proposed for Bayesian estimation algorithm and the weighted estimation of normal distribution parameters is provided. And the statistical diversity between the weighted and classical methods is quantified, and upper bound of the calculation errors caused by prior distortion is deduced for mixture of finite normal distributions. Taking the estimation of the dispersion of the guided weapon impact points as an example, the conclusion is drawn that our method is credible.
\end{abstract}

\section{Introduction}

Circular Error Probable (CEP) is a common measure used in the evaluation test of guidance weapon systems, which can integrate precision with dispersion to assess the hit accuracy of projectiles [1]. In traditional CEP assessment process, the impact point deviation is assumed to follow bivariate normal distribution, and based on the formula simplified by decorrelation coordinate transformation, the MLE (maximum likelihood estimator) of normal distribution parameters are calculated by specific samples, which are employed in the forthcoming point estimation, confidence bounds computation, and hypothesis testing of CEP [2].

There are two restrictions for above CEP evaluation procedure. One is the concrete computational difficulty. It is hard to solve the complex CEP equation precisely, so some approximative expressions are adopted in practical applications with different assumptions [2]. In [3] 12 methods of calculating CEP are summarized and classified into three categories: univariate parameterization, binary computation, and numerical integration.

Another limitation is adaptability of the estimate process. The basis of bivariate normal distribution assumption is the central-limit theorem and engineering experience accumulated for ages [4]. Compatibility and normality test are called for the hypothesis based on a certain confidence level. But the assumption is not suitable for complicated data, which demands expanding the ranges of CEP application to obtain more adaptable and reasonable results of hit accuracy $[1,2$, $5,6]$. A bias-corrected estimator of CEP is provided based on Cornish-Fisher expansion in [2]. And in [5, 6], CEP is extended to ACEP (Area Circular Error Probability) to overcome the disadvantage that CEP is not combined with the self-characteristics of surface targets. Reference [7] considers the case that missile impact samples is not i.i.d. and provides a Bayesian procedure for estimating CEP.

For INS (Inertial Navigation System)/SAR (Synthetic Aperture Radar) integrated system, the system biases of impact point errors are quite distinct in diverse experiment conditions or scene features. Under the circumstances classical CEP evaluation is not applicable because it is tough to treat various samples as generated by the same normal population. In addition, the stochastic quality of scene matching also increases computational difficulty. In order to use the samples following diverse populations to assess accuracy of projectiles, this paper provides the CEP evaluation procedure under the circumstance that impact samples are not i.i.d. data. In Section 2, classical CEP is generalized to the CEP of multiconditional probability based on nonparametric mixture model. Then specific computational method for mixed CEP model is designed in Section 3, which includes simplification of general equation, point, and 
interval estimation. The algorithms of mixed and classical CEP are compared and analyzed in Section 4. In Section 5 some simulation experiments are implemented, the results of which indicate that the mixed CEP algorithm is more reasonable than traditional one under the circumstances that diversity of accuracy samples is obvious.

\section{Modeling of Bayes Model of Scene Matching of Multiconditional Probability}

2.1. Classical CEP Definition. The definition of CEP is distinct in kinds of literature, and a common one is adopted in this paper. CEP is the radius of a circle, where center is target and the probability of an impact point inside is 50\% [1]. The corresponding mathematical description is as follows. Establish a rectangular coordinate system centered on target with $(0,0)$, and assume the deviation of downrange and crossrange noted as $X$ and $Z$, respectively, both follows normal distribution; then the probability density function of $\eta=$ $(X, Z)^{T}$ is

$$
\begin{aligned}
& f(x, z)=\frac{1}{2 \pi \sigma_{x} \sigma_{z} \sqrt{1-\rho^{2}}} \\
& \cdot \exp \left\{-\frac{1}{2\left(1-\rho^{2}\right)}\left[\frac{\left(x-\mu_{x}\right)^{2}}{\sigma_{x}^{2}}\right.\right. \\
& \left.\left.-\frac{2 \rho\left(x-\mu_{x}\right)\left(z-\mu_{z}\right)}{\sigma_{x} \sigma_{z}}+\frac{\left(z-\mu_{z}\right)^{2}}{\sigma_{z}^{2}}\right]\right\},
\end{aligned}
$$

where $\sigma_{x}, \sigma_{z}$ are standard deviations of $X, Z ; \mu_{x}, \mu_{z}$ are means of $X, Z ; \rho$ is correlation coefficient of $X$ and $Z$, such that $0 \leq$ $|\rho|<1$. Then $R$ in the following formula is the CEP of $\eta=$ $(X, Z)^{T}$

$$
\iint_{x^{2}+z^{2} \leqslant R^{2}} f(x, z) d x d z=0.5
$$

where $f(x, z)$ is the same as (1). And (2) is called the general form of CEP equation.

Suppose $\left\{\mathbf{u}_{i} \mid \mathbf{u}_{i}=\left(x_{i}, z_{i}\right)^{T}\right\}_{i=1}^{S}$ are the impact deviation samples to be used. In previous CEP algorithms they are utilized to calculate $\sigma_{x}, \sigma_{z}, \mu_{x}, \mu_{z}, \rho$ in (1), which are employed to estimate $R$ by numerical integration or approximate approaches in (2). But samples collected in different test environments and influenced by complicate factors such as scene features are distinct, which is against the assumption of same population, and make the traditional CEP evaluation invalid. Under this condition we propose the mixed CEP model and algorithm of multiconditional probability.

2.2. Bayes Nonparametric Mixture Model. The Bayes mixture model combined with random density function is as follows:

$$
f(y)=\int K(y ; \theta) d P(\theta)
$$

where $K(y ; \theta)$ is density function with different parameters $\theta$. And $P(\theta)$ is random density function as the following discrete form usually:

$$
P(d \theta)=\sum_{l=1}^{\infty} \omega_{l} \delta_{\theta_{l}}(d \theta),
$$

where $\left(\omega_{l}, \theta_{l}\right)_{l=1}^{\infty}$ is prior information and $\delta_{\theta_{l}}$ is characteristic function with various $\theta$.

2.3. Mixed CEP Model for Scene Matching of Multiconditional Probability. Define mixed CEP as the radius of a circle centered on the target, such that the probability of an impact point inside the circle is $50 \%$, while impact deviation does not follow the same bivariate normal population. For INS/SAR integrated navigation system, the impact biases are influenced by scene matching, of which the error sources such as scene features, types, and matching number are quite distinct and follow diverse populations [8]. In order to assess hit accuracy of the system by mixed CEP, modeling for scene matching is essential beforehand. Since the types and occurring probability of scene in one test are unknown before the experiment implement, they can be regarded as random variables while modeling based on Bayes nonparametric model frame.

Suppose synthetical test conditions of scene matching are classified into $N$ types denoted as $h_{1}, h_{2}, \ldots, h_{N}$ of which occurring probability is $p\left(h_{1}\right), p\left(h_{2}\right), \ldots, p\left(h_{N}\right) ; p\left(h_{i}\right)(i=$ $1,2, \ldots, N)$ are random variables in $[0,1]$, such that

$$
\sum_{k=1}^{N} p\left(h_{k}\right)=1 .
$$

Consider the conditional probability density function of impact errors on $h_{k}$ is normal as follows:

$$
\begin{aligned}
& f_{k}\left(x, z \mid h_{k}\right)=\frac{1}{2 \pi \sigma_{x_{k}} \sigma_{z_{k}} \sqrt{1-\rho_{k}^{2}}} \\
& \cdot \exp \left\{-\frac{1}{2\left(1-\rho_{k}^{2}\right)}\left[\frac{\left(x-\mu_{x_{k}}\right)^{2}}{\sigma_{x_{k}}^{2}}\right.\right. \\
& \left.\left.-\frac{2 \rho_{k}\left(x-\mu_{x_{k}}\right)\left(z-\mu_{z_{k}}\right)}{\sigma_{x_{k}} \sigma_{z_{k}}}+\frac{\left(z-\mu_{z_{k}}\right)^{2}}{\sigma_{z_{k}}^{2}}\right]\right\},
\end{aligned}
$$

where $\sigma_{x_{k}}, \sigma_{z_{k}}$ are standard deviations of $X, Z$ on condition $h_{k} ; \mu_{x_{k}}, \mu_{z_{k}}$ are means of $X, Z$ on condition $h_{k} ; \rho_{k}$ is correlation coefficient of $X, Z$ on condition $h_{k}$, such that $0 \leq\left|\rho_{k}\right|<1$. Then the joint probability density function of impact errors is

$$
f(x, z)=\int f_{k}\left(x, z \mid h_{k}\right) p\left(h_{k}\right) d h_{k}
$$

The discrete form is

$$
f(x, z)=\sum_{k=1}^{N} p\left(h_{k}\right) f_{k}\left(x, z \mid h_{k}\right) .
$$


Substitute (8) for $f(x, z)$ in (2), and $R$ in the following equation is the mixed CEP of multiconditional probability

$$
\iint_{x^{2}+z^{2} \leqslant R^{2}} \sum_{k=1}^{N} p\left(h_{k}\right) f_{k}\left(x, z \mid h_{k}\right) d x d z=0.5 .
$$

\section{Mixed CEP Algorithm of Multiconditional Probability}

3.1. Simplification of General CEP Equation. For condition $h_{k}$, take orthogonal transformation for (6) as follows:

$$
\left(\begin{array}{l}
u \\
v
\end{array}\right)=\left(\begin{array}{cc}
\cos \xi_{k} & \sin \xi_{k} \\
-\sin \xi_{k} & \cos \xi_{k}
\end{array}\right)\left(\begin{array}{l}
x \\
z
\end{array}\right),
$$

where

$$
\xi_{k}=\frac{1}{2} \operatorname{tg}^{-1} \frac{2 \rho_{k} \sigma_{x_{k}} \sigma_{z_{k}}}{\sigma_{x_{k}}{ }^{2}-\sigma_{z_{k}}^{2}} .
$$

Then $(X, Z)$ is transformed to $(U, V)$, of which $U$ and $V$ are independent of condition $h_{k}$. And probability density function of $(U, V)$ on condition $h_{k}$ is

$$
\begin{aligned}
& f_{k}\left(u, v \mid h_{k}\right)=\frac{1}{2 \pi \sigma_{u_{k}} \sigma_{v_{k}}} \\
& \cdot \exp \left\{-\frac{1}{2}\left[\frac{\left(u-\mu_{u_{k}}\right)^{2}}{\sigma_{u_{k}}^{2}}+\frac{\left(v-\mu_{v_{k}}\right)^{2}}{\sigma_{v_{k}}^{2}}\right]\right\},
\end{aligned}
$$

where $\sigma_{u_{k}}, \sigma_{v_{k}}$ are standard deviations of $(U, V)$ on condition $h_{k} ; \mu_{u_{k}}, \mu_{v_{k}}$ are means of $(U, V)$ on condition $h_{k}$. Then (9) is turned into

$$
\iint_{u^{2}+v^{2} \leqslant R^{2}} \sum_{k=1}^{N} p\left(h_{k}\right) f_{k}\left(u, v \mid h_{k}\right) d u d v=0.5 .
$$

Take polar coordinate transformation as follows:

$$
\begin{aligned}
& u=r \cos \varphi \\
& v=r \sin \varphi,
\end{aligned}
$$

and then (13) is simplified from the form of double integral to the following quadratic integral:

$$
\begin{aligned}
& \frac{1}{2 \pi} \int_{0}^{R} \int_{0}^{2 \pi} r \sum_{k=1}^{N} p\left(h_{k}\right) \cdot c_{k} \\
& \quad \cdot \exp \left[-b_{k} r^{2}+a_{k} r^{2} \cos 2 \varphi+r\left(\frac{\mu_{u_{k}}}{\sigma_{u_{k}}^{2}} \cos \varphi+\frac{\mu_{v_{k}}}{\sigma_{v_{k}}^{2}} \sin \varphi\right)\right] d \varphi d r \\
& \quad=0.5,
\end{aligned}
$$

where

$$
\begin{aligned}
& a_{k}=\frac{1}{4}\left(\frac{1}{\sigma_{v_{k}}^{2}}-\frac{1}{\sigma_{u_{k}}^{2}}\right) \\
& b_{k}=\frac{1}{4}\left(\frac{1}{\sigma_{v_{k}}^{2}}+\frac{1}{\sigma_{u_{k}}^{2}}\right) \\
& c_{k}=\frac{1}{\sigma_{u_{k}} \sigma_{v_{k}}} \exp \left\{-\frac{1}{2}\left[\left(\frac{\mu_{u_{k}}}{\sigma_{u_{k}}}\right)^{2}+\left(\frac{\mu_{v_{k}}}{\sigma_{v_{k}}}\right)^{2}\right]\right\} .
\end{aligned}
$$

3.2. Mixed CEP Point Estimates of Multiconditional Probability. Suppose there are $N$ types of impact error samples $\left\{\mathbf{u}_{i}^{(k)} \mid\right.$ $\left.\mathbf{u}_{i}^{(k)}=\left(x_{i}^{(k)}, z_{i}^{(k)}\right)^{T}\right\}_{i=1}^{n_{k}},(k=1,2, \ldots, N)$ such that $\sum_{i=1}^{N} n_{k}=$ $S$. Assume $\left\{\mathbf{u}_{i}^{(k)}\right\}_{i=1}^{n_{k}}$ follow normal populations $N\left(\mu_{k}, \Sigma_{k}\right)$, respectively, then the estimates of normal parameters are as follows:

$$
\begin{aligned}
& \widehat{\mu}_{u_{k}}=\widehat{\mu}_{x_{k}} \cos \widehat{\xi}_{k}+\widehat{\mu}_{z_{k}} \sin \widehat{\xi}_{k} \\
& \widehat{\mu}_{v_{k}}=\widehat{\mu}_{z_{k}} \cos \widehat{\xi}_{k}-\widehat{\mu}_{x_{k}} \sin \widehat{\xi}_{k} \\
& \widehat{\sigma}_{u_{k}} \\
& =\sqrt{\widehat{\sigma}_{x_{k}}^{2} \cos ^{2} \widehat{\xi}_{k}+\widehat{\sigma}_{z_{k}}^{2} \sin ^{2} \widehat{\xi}_{k}+2 \widehat{\rho}_{k} \widehat{\sigma}_{x_{k}} \widehat{\sigma}_{z_{k}} \cos \widehat{\xi}_{k} \sin \widehat{\xi}_{k}} \\
& \widehat{\sigma}_{v_{k}} \\
& =\sqrt{\widehat{\sigma}_{z_{k}}^{2} \cos ^{2} \widehat{\xi}_{k}+\widehat{\sigma}_{x_{k}}^{2} \sin ^{2} \widehat{\xi}_{k}-2 \widehat{\rho}_{k} \widehat{\sigma}_{x_{k}} \widehat{\sigma}_{z_{k}} \cos \widehat{\xi}_{k} \sin \widehat{\xi}_{k}},
\end{aligned}
$$

where

$$
\begin{aligned}
& \widehat{\mu}_{x_{k}}=\frac{1}{n_{k}} \sum_{i=1}^{n_{k}} x_{i}^{(k)} \\
& \widehat{\mu}_{z_{k}}=\frac{1}{n_{k}} \sum_{i=1}^{n_{k}} z_{i}^{(k)} \\
& \widehat{\sigma}_{x_{k}}=\sqrt{\frac{1}{n_{k}-1} \sum_{i=1}^{n_{k}}\left(x_{i}^{(k)}-\widehat{\mu}_{x_{k}}\right)^{2}} \\
& \widehat{\sigma}_{z_{k}}=\sqrt{\frac{1}{n_{k}-1} \sum_{i=1}^{n_{k}}\left(z_{i}^{(k)}-\widehat{\mu}_{z_{k}}\right)^{2}}, \\
& \widehat{\rho}_{k}=\frac{\sum_{i=1}^{n_{k}}\left[\left(x_{i}^{(k)}-\widehat{\mu}_{x_{k}}\right)\left(z_{i}^{(k)}-\widehat{\mu}_{z_{k}}\right)\right]}{\sqrt{\left[\sum_{i=1}^{n_{k}}\left(x_{i}^{(k)}-\widehat{\mu}_{x_{k}}\right)^{2}\right]\left[\sum_{i=1}^{n_{k}}\left(z_{i}^{(k)}-\widehat{\mu}_{z_{k}}\right)^{2}\right]}}, \\
& \widehat{\xi}_{k}=\frac{1}{2} \operatorname{tg}^{-1} \frac{2 \widehat{\rho}_{k} \widehat{\sigma}_{x_{k}} \widehat{\sigma}_{z_{k}}}{\widehat{\sigma}_{x_{k}}^{2}-\widehat{\sigma}_{z_{k}}^{2}} \text {. }
\end{aligned}
$$

Suppose prior information of text conditions is explicit; that is,

$$
E\left[p\left(h_{k}\right)\right]=p_{k}
$$

where $p_{k}$ are all constants, such that

$$
\sum_{k=1}^{N} p_{k}=1
$$

Substitute $\widehat{\mu}_{u_{k}}, \widehat{\mu}_{v_{k}}, \widehat{\sigma}_{u_{k}}, \widehat{\sigma}_{v_{k}}, p_{k}$ for $\mu_{u_{k}}, \mu_{v_{k}}, \sigma_{u_{k}}, \sigma_{v_{k}},\left[p\left(h_{k}\right)\right]$ in (15); then mixed CEP can be calculated approximately with numerical integration and bisection algorithm.

3.3. Mixed CEP Interval Estimation of Multiconditional Probability. Bootstrap method $[9,10]$ is called for calculating 
mixed CEP confidence interval estimation of multiconditional probability here. Suppose $\widehat{R}$ is the plug-in point estimator of $R$. $C_{\alpha}$ denote $\alpha$ quantile of $R$; that is, $P\left(R \leq C_{\alpha}\right)=$ $\alpha$, and corresponding estimator is $\widehat{C}_{\alpha}$. Then the calculation formulas of confidence interval $\left[R_{b u}, R_{b l}\right]$ and confidence upper bound are given as follows:

$$
\begin{aligned}
R_{b u} & =\widehat{C}_{1-\alpha / 2}, \\
R_{b l} & =\widehat{C}_{\alpha / 2}, \\
R_{b} & =\widehat{C}_{1-\alpha},
\end{aligned}
$$

where $\widehat{C}_{1-\alpha / 2}, \widehat{C}_{\alpha / 2}, \widehat{C}_{\alpha}$ are approximately computed by the following steps.

(1) Calculate $\widehat{\mu}_{u_{k}}, \widehat{\mu}_{v_{k}}, \widehat{\sigma}_{u_{k}}, \widehat{\sigma}_{v_{k}}$ by samples $\left\{\left(x_{i}^{(k)}, z_{i}^{(k)}\right)\right\}_{i=1}^{n_{k}}$ in $(17)(k=1,2, \ldots, N)$.

(2) Resample $M$ times from bivariate normal distribution $N\left(\left(\widehat{\mu}_{u_{k}}, \widehat{\mu}_{v_{k}}\right)^{T}, \operatorname{diag}\left(\widehat{\sigma}_{u_{k}}^{2}, \widehat{\sigma}_{v_{k}}^{2}\right)\right)$ with size $n_{k}$, respectively $(k=1,2, \ldots, N)$. Then compute $M$ point estimators of $R$ by Section 3.2, and sort them from lowest as $\widehat{R}^{(1)}$, $\widehat{R}^{(2)}, \ldots, \widehat{R}^{(M)}$, where $1000 \leqslant M \leqslant 3000$.

(3) The INT[(1 - $\alpha / 2) M]$ th, INT $[(\alpha / 2) M]$ th, and INT $[\alpha M]$ th one of the sequences $\widehat{R}^{(1)}, \widehat{R}^{(2)}, \ldots, \widehat{R}^{(M)}$ are $\widehat{C}_{1-\alpha / 2}, \widehat{C}_{\alpha / 2}, \widehat{C}_{\alpha}$, where INT[·] is rounding operator.

\section{Performance Analysis of the Algorithm}

4.1. Comparison of Statistical Properties. The statistical properties of mixed CEP algorithm are compared with the classical one for the bivariate mixed population of two normal distributions as an example. According to Section 3.1, the relationship between the downrange and cross-range error can be decorrelated by coordinate transformation. Therefore the following discussion focuses only on the independent case.

Suppose there are $N$ types of impact error samples $\left\{\mathbf{u}_{i}^{(k)} \mid\right.$ $\left.\mathbf{u}_{i}^{(k)}=\left(x_{i}^{(k)}, z_{i}^{(k)}\right)^{T}\right\}_{i=1}^{n_{k}}(k=1,2, \ldots N)$ such that $\sum_{i=1}^{N} n_{k}=$ $S$. Assume $\left\{\mathbf{u}_{i}^{(k)}\right\}_{i=1}^{n_{k}}$ follow the normal population $N\left(\mu_{k}, \Sigma_{k}\right)$, which are under the conditions $h_{k}$, respectively. Then the joint probability density function of impact errors is as (8), where $f_{k}\left(x, z \mid h_{k}\right)$ is probability density function of $N\left(\mu_{k}, \Sigma_{k}\right)(k=$ $1,2, \ldots, N)$. And the estimates of normal parameters are as follows:

$$
\begin{aligned}
& \widehat{\mu}_{k}=\left(\begin{array}{l}
\widehat{\mu}_{x_{k}} \\
\widehat{\mu}_{z_{k}}
\end{array}\right), \\
& \widehat{\Sigma}_{k}=\left(\begin{array}{cc}
\widehat{\sigma}_{x_{k}}^{2} & 0 \\
0 & \widehat{\sigma}_{z_{k}}^{2}
\end{array}\right) .
\end{aligned}
$$

If the prior probabilities of test experiments are fixed as $E\left[p\left(h_{k}\right)\right]=p_{k}\left(0 \leqslant p_{k} \leqslant 1\right)$ and populations $N\left(\mu_{k}, \Sigma_{k}\right)$ are independent, then the mean and variance estimates of mixed population $f(x, z)$ will be

$$
\begin{aligned}
\widehat{E}[\eta] & =\sum_{k=1}^{N} p_{k} \widehat{\mu}_{k}, \\
\widehat{\operatorname{Var}}[\eta] & =\sum_{k=1}^{N} p_{k}^{2} \widehat{\Sigma}_{k} .
\end{aligned}
$$

If the impact error samples $\left\{\mathbf{u}_{i}^{(k)} \mid \mathbf{u}_{i}^{(k)}=\left(x_{i}^{(k)}, z_{i}^{(k)}\right)^{T}\right\}_{i=1}^{n_{k}}$ $(k=1,2, \ldots, N)$ are following the same normal population $N\left(\mu_{s}, \Sigma_{s}\right)$ the estimates of normal parameters are

$$
\begin{aligned}
& \widehat{\mu}_{s}=\left(\begin{array}{l}
\widehat{\mu}_{x_{s}} \\
\widehat{\mu}_{z_{s}}
\end{array}\right)=\frac{1}{S} \sum_{k=1}^{N} n_{k} \widehat{\mu}_{k}, \\
& \widehat{\Sigma}_{s}=\frac{1}{S-1} \sum_{k=1}^{N} \sum_{i=1}^{n_{k}}\left(\begin{array}{cc}
x_{i}^{(k)}-\widehat{\mu}_{x_{s}} & 0 \\
0 & z_{i}^{(k)}-\widehat{\mu}_{z_{s}}
\end{array}\right)^{2} .
\end{aligned}
$$

Thus the errors of single normal population approximated to the bivariate mixed population of two normal distributions are

$$
\begin{aligned}
& \Delta_{E}=\widehat{\mu}_{s}-\widehat{E}[\eta]=\sum_{k=1}^{N}\left(\frac{n_{k}}{S}-p_{k}\right) \widehat{\mu}_{k}, \\
& \Delta_{D}=\widehat{\Sigma}_{s}-\widehat{\operatorname{Var}}[\eta]=\sum_{k=1}^{N}\left(\frac{n_{k}-1}{S-1}-p_{k}^{2}\right) \widehat{\Sigma}_{k}+\varepsilon,
\end{aligned}
$$

where

$$
\begin{aligned}
\varepsilon & =\frac{2}{S-1} \\
& \cdot \sum_{k=1}^{N} \sum_{i=1}^{n_{k}}\left(\begin{array}{cc}
\left(x_{i}^{(k)}-\widehat{\mu}_{x_{k}}\right)\left(\widehat{\mu}_{x_{k}}-\widehat{\mu}_{x_{s}}\right) & 0 \\
0 & \left(z_{i}^{(k)}-\widehat{\mu}_{z_{k}}\right)\left(\widehat{\mu}_{z_{k}}-\widehat{\mu}_{z_{s}}\right)
\end{array}\right) \\
& =0 .
\end{aligned}
$$

By (25), it indicates that the means of two algorithms are the same when $\widehat{\mu}_{k}=\mathbf{0}$ or $p_{k}=n_{k} / S, \forall k=1,2, \ldots, N$. But as is shown in (26), variances of two algorithms are distinct under these circumstances.

4.2. Influence of Prior Distortion to Mixed CEP Errors. The selection of prior information is very influential to Bayesian posterior estimate. If the prior is not accurate, the error of assessment results may be very large. Here the influence of prior distortion to mixed CEP calculation is discussed for finite mixture model as sensitivity analysis.

Let

$$
\iint_{x^{2}+z^{2} \leqslant R^{2}} f_{k}\left(x, z \mid h_{k}\right) d x d z=0.5,
$$

and note the solutions of (9) and (28) are $R$ and $R_{k}(k=$ $1,2, \ldots, N)$. Suppose $R_{i} \leqslant R_{j}(i<j, i, j=1,2, \ldots, N)$ without loss of generality; then we have the following equation:

$$
R_{1} \leqslant R \leqslant R_{N} .
$$




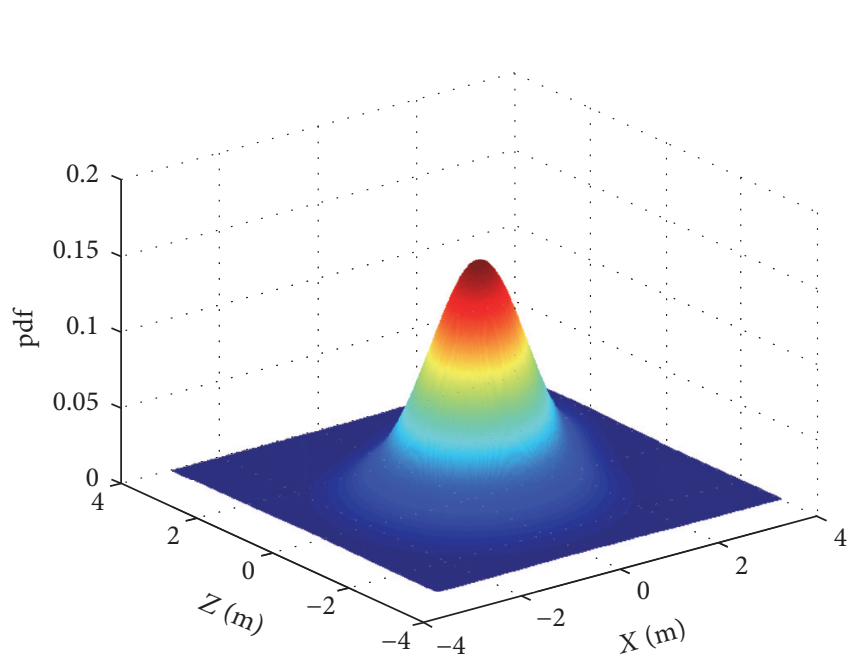

(a) 3 dimensions

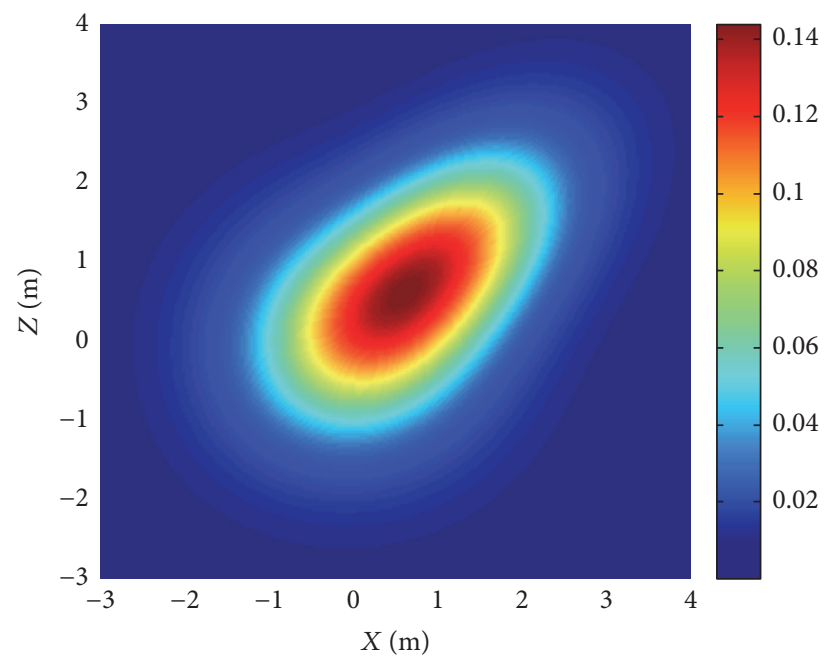

(b) 2 dimensions

FIgURe 1: Probability density function of two normal mixture populations.

In the equation above $R_{1}$ and $R_{N}$ are the lower and upper bound of two normal mixture population, of which the corresponding values of the prior weights $P_{w}=\left(p\left(h_{1}\right)\right.$, $\left.p\left(h_{2}\right), \ldots, p\left(h_{N}\right)\right)^{T}$ are $(1,0, \ldots, 0)^{T}$ and $(0,0, \ldots, 1)^{T}$, respectively. It indicates that the CEP response reaches extremums at the interval boundaries of prior weights. Thus we have the proposition as follows.

Proposition 1. Suppose random vector follows the mixture distribution of which probability density function is the same as (8). Prior weights $P_{w}=\left(p\left(h_{1}\right), p\left(h_{2}\right), \ldots, p\left(h_{N}\right)\right)^{T}$ is assumed as a random vector in $[0,1]^{N}$, such that $E\left[p\left(h_{k}\right)\right]=p_{k}(k=$ $1,2, \ldots, N)$, and $\sum_{1}^{N} p\left(h_{k}\right)=\sum_{1}^{N} p_{k}=1$. Note $\widehat{P}_{w}$ is the estimate of $P_{w}$, and $\triangle R$ is the error of mixed CEP with $\widehat{P}_{w}$. Then

$$
\sup _{\widehat{P}_{w} \in[0,1]^{N},\left\|\widehat{P}_{w}\right\|=1} \Delta R\left(\widehat{P}_{w}\right) \leqslant\left|\max _{k=1,2, \ldots, N}\left\{R_{k}\right\}-R_{0}\right|,
$$

where $R_{0}$ is the solution of (9) with $p\left(h_{k}\right)=p_{k}(k=$ $1,2, \ldots, N)$ and $R_{k}$ denotes the solution of (9) with $p\left(h_{k}\right)=1$ and $p\left(h_{j}\right)=0(k=1,2, \ldots, N, j \neq k)$.

\section{Numerical Experiments}

5.1. Example 1. Suppose synthetical test conditions of scene matching are $h_{1}$ and $h_{2}$ with $E\left[p\left(h_{1}\right)\right]=0.4$ and $E\left[p\left(h_{2}\right)\right]=$ 0.6. And assume the impact errors $(X, Z)$ follow normal distributions $N\left(\left(\begin{array}{l}0 \\ 0\end{array}\right),\left(\begin{array}{cc}1 & 0.01 \\ 0.01 & 1\end{array}\right)\right)$ and $N\left(\left(\begin{array}{l}1 \\ 1\end{array}\right),\left(\begin{array}{cc}1 & 0.5 \\ 0.5 & 1\end{array}\right)\right)$ under the conditions $h_{1}$ and $h_{2}$, respectively. Then the probability density function of two normal mixture population is shown in Figure 1.

As displayed in Figure 1, the mixed population is still unimodal, of which the theoretical, classical, and mixed CEP are $1.414 \mathrm{~m}, 1.215 \mathrm{~m}$, and $1.420 \mathrm{~m}$. Take sample from normal populations 1 and 2 with sizes 20 and 30, respectively, of which the dispersion is shown in Figure 2. In Figure 2 only 8 samples

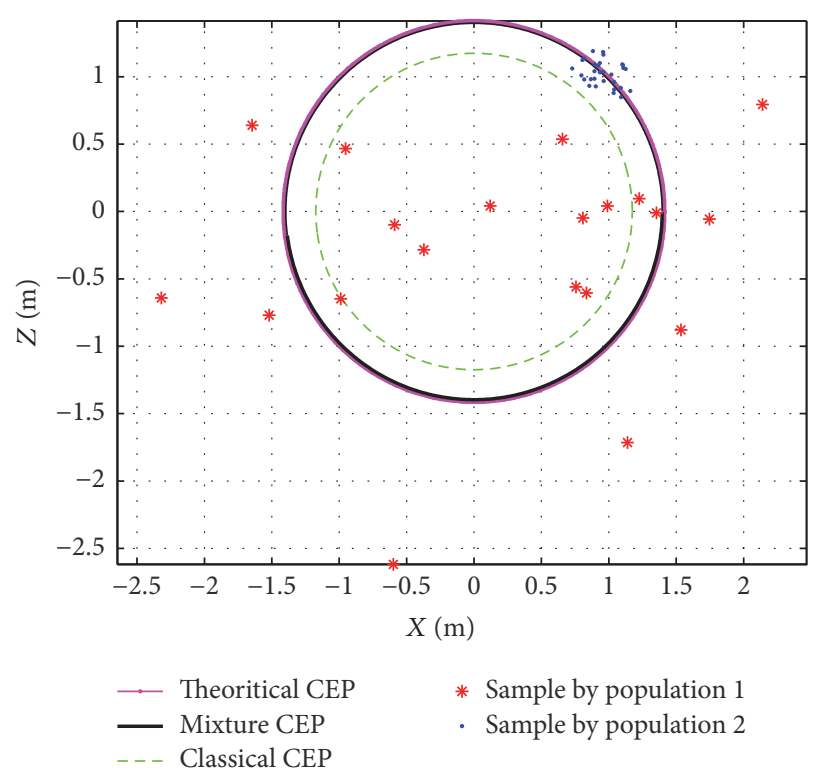

FIGURE 2: Sample dispersion and comparison of CEP by different algorithms.

(16\%) impact in the circle of classical CEP, which does not accord with the definition of CEP. Meanwhile there are 28 samples (56\%) in the circle of mixed CEP, which illustrates that the mixed CEP calculation algorithm in this paper is more adaptable and accurate contrasted with theoretical and classical CEP under the circumstance.

In order to reduce the influence of random factors to CEP calculation result, resample 10 and 100 times under the circumstance that weights, sample size, and parameters of two normal populations are fixed, and the averages of corresponding computing results are shown in Table 1.

In Table 1, it indicates that the average precision of mixed CEP algorithm is larger than classical one on the condition 
TABLE 1: Contrast average CEP mixed with classical algorithms by resampling.

\begin{tabular}{lccccc}
\hline \multirow{2}{*}{ Sampling times } & \multicolumn{2}{c}{ CEP algorithms } & \multicolumn{2}{c}{ Samples in CEP circle } & Percentage of more accurate mixed CEP \\
& Mixed & Classical & Mixed & Classical & $100 \%$ \\
1 & 1.420 & 1.215 & $56 \%$ & $16 \%$ & $100 \%$ \\
10 & 1.357 & 1.176 & $47 \%$ & $24 \%$ & $77 \%$ \\
\hline
\end{tabular}

TABLE 2: CEP and corresponding errors with different prior information.

\begin{tabular}{lccc}
\hline$\widehat{p}_{1}$ & $\Delta \widehat{p}_{1}$ & $\widehat{R}$ & $\Delta \widehat{R}$ \\
\hline 0 & 0.4 & 1.633 & 0.219 \\
0.1 & 0.3 & 1.573 & 0.160 \\
0.2 & 0.2 & 1.517 & 0.104 \\
0.3 & 0.1 & 1.464 & 0.050 \\
0.4 & 0 & 1.414 & 0 \\
0.5 & 0.1 & 1.367 & -0.047 \\
0.6 & 0.2 & 1.323 & -0.090 \\
0.7 & 0.3 & 1.283 & -0.131 \\
0.8 & 0.4 & 1.245 & -0.169 \\
0.9 & 0.5 & 1.210 & -0.204 \\
1 & 0.6 & 1.177 & -0.236 \\
\hline
\end{tabular}

TABLE 3: Value of impact errors.

\begin{tabular}{|c|c|c|c|c|c|c|c|c|c|c|}
\hline \multirow{2}{*}{ Test condition } & \multirow{2}{*}{ Direction } & \multicolumn{9}{|c|}{ Test data (m) } \\
\hline & & 1 & 2 & 3 & 4 & 5 & 6 & 7 & 8 & 9 \\
\hline \multirow{2}{*}{1} & $X$ & 0.538 & 1.834 & -2.259 & 0.862 & 0.319 & -1.308 & -0.434 & 0.343 & 3.578 \\
\hline & $Z$ & 2.769 & -1.350 & 3.035 & 0.725 & -0.063 & 0.715 & -0.205 & -0.124 & 1.490 \\
\hline \multirow{2}{*}{2} & $X$ & 0.641 & 0.642 & 0.567 & 0.379 & 0.572 & 0.663 & 0.549 & 0.604 & 0.573 \\
\hline & $Z$ & 0.470 & 0.529 & 0.421 & 0.589 & 0.385 & 0.393 & 0.419 & 0.206 & 0.644 \\
\hline \multirow{2}{*}{3} & $X$ & 1.033 & 0.925 & 1.137 & 0.829 & 0.990 & 0.976 & 1.032 & 1.031 & 0.914 \\
\hline & $Z$ & 0.997 & 0.984 & 1.063 & 1.109 & 1.111 & 0.914 & 1.008 & 0.879 & 0.889 \\
\hline
\end{tabular}

TABLE 4: Actual and prior mean of probability of test conditions.

\begin{tabular}{lcc}
\hline $\begin{array}{l}\text { Test } \\
\text { condition }\end{array}$ & $\begin{array}{c}\text { Mean of real } \\
\text { probability }\end{array}$ & $\begin{array}{c}\text { Prior mean of } \\
\text { probability }\end{array}$ \\
\hline 1 & $1 / 3$ & $3 / 10$ \\
2 & $1 / 3$ & $3 / 10$ \\
3 & $1 / 3$ & $2 / 5$ \\
\hline
\end{tabular}

of resampling, and the probability when mixed CEP is more precise than typical one is large (about $77 \%$ in resampling 100 times) under the circumstance that samples are fixed. In addition, viewed from the perspective of containing samples, the proportion of samples in the circle of mixed CEP much more comply with the CEP definition, of which the average is about $44 \%$ in resampling times. Meanwhile the corresponding value of classical CEP circle is far from the ideal $50 \%$, of which the average is about $21 \%$.
5.2. Example 2. The influence of prior distortion to mixed CEP errors for two normal mixture population is discussed in this subsection, of which the related parameters are the same as Section 5.1. In this case the input of prior parameter is only $\widehat{p}_{1} \in[0,1]$, while $\widehat{p}_{2}$ can be replaced by $\widehat{p}_{2}=1-\widehat{p}_{1}$. So the relationship between $\widehat{p}_{1}$ and mixed CEP can be expressed as the following equation:

$$
\int_{x^{2}+z^{2} \leq R^{2}}\left[f_{2}+\widehat{p}_{1}\left(f_{1}-f_{2}\right)\right] d x d z=0.5
$$

where $f_{1}$ and $f_{2}$ denote density functions of normal distribution 1 and 2 . Suppose $\widehat{p}_{1}=0.4$ is the real prior information, and the exact mixed CEP is $1.414 \mathrm{~m}$. Then CEP and corresponding errors are calculated on the conditions of $\widehat{p}_{1}=0.1 k(k=0,1, \ldots, 10)$, which are shown in Table 2 .

As is shown in Table 2, the CEP error $\Delta \widehat{R}$ increases as the prior error $\Delta \widehat{p}_{1}$ increases. And we can see the maximum of CEP under prior distortion is achieved by the boundary 
TABLE 5: CEP under different assumptions of the population.

\begin{tabular}{|c|c|c|c|c|c|}
\hline \multirow{2}{*}{ Assumptions of the population } & \multirow{2}{*}{ CEP point estimate } & \multicolumn{2}{|c|}{ CEP confidence upper bound } & \multicolumn{2}{|c|}{ CEP confidence interval } \\
\hline & & Level 0.9 & Level 0.8 & Level 0.9 & Level 0.8 \\
\hline $\begin{array}{l}\text { Single } \\
\text { Sing }\end{array}$ & 1.416 & 1.595 & 1.527 & {$[1.220,1.665]$} & {$[1.261,1.595]$} \\
\hline Mixture with prior distortion & 1.356 & 1.404 & 1.383 & {$[1.299,1.421]$} & {$[1.314,1.404]$} \\
\hline Mixture without prior distortion & 1.340 & 1.390 & 1.369 & {$[1.281,1.410]$} & {$[1.293,1.390]$} \\
\hline
\end{tabular}

point $\widehat{p}_{1}=0.4$, which is in accordance with Proposition 1 . In addition, we can conclude that prior distortion can deviate the mixed CEP from theoretic value indeed, but the upper bound of errors is estimable, which is quantified in Proposition 1.

5.3. Example 3. The assessment of CEP in the case of small samples is discussed in this subsection. Assume 9 impact error samples are obtained after a group of flight tests and divided into 3 classes under corresponding conditions, which is shown in Table 3. In addition, real and prior mean of probability of test conditions are given in Table 4 . Then the CEP point estimators and confidence intervals can be calculated after resampling 1000 times under the different assumptions, which is shown in Table 5.

The CEP confidence upper bounds under different assumptions are compared and analyzed here. As is shown in Table 5, the assessment results by mixed CEP method are closer to the mixed CEP of accurate prior information compared with classical single algorithm, even with the distorted prior. It illustrates that the adaptive ability of mixed CEP algorithm is promising in the case of small samples.

\section{Conclusions}

We have investigated the problem that classical CEP method may be unreasonable and may give inaccurate results for the impact samples following different populations. For purpose of addressing it, the mixed CEP is modeled in the context that the errors caused by scene matching follows diverse populations, and corresponding algorithms of point estimator and confidence interval estimation are designed. Taking mixed population of two normal distributions as an example, the statistical properties of traditional and mixed CEP algorithm are compared, and it concludes that prior distortion can deviate the mixed CEP from theoretic value indeed, but the upper bound of errors is estimable.

In numerical experiments, CEP calculated by mixed algorithm is close to theoretical value in the case of the population mixing two normal distributions, while traditional CEP method is unreasonable and unprecise, which illustrates that the mixed CEP algorithm designed in this paper is adaptable under the circumstance. In addition, the response relationship of prior parameters and CEP is analyzed in the case, which verifies Proposition 1 from the perspective of statistical simulation. And on the condition of small samples with mixture population of three normal distributions, the confidence upper bounds of mixed CEP with real and biased prior parameters are calculated, which indicates that the mixed CEP algorithm is credible for small samples.

In the case of impact samples following the mixed population of finite normal distributions, the mixed CEP algorithm proposed in this paper is credible. However, the effect of the method to other types of mixture models is not explicit, which is what we aim to investigate furthermore.

\section{Competing Interests}

The authors declare that they have no competing interests.

\section{Acknowledgments}

This research is partially supported by the program for New Century Excellent Talents in University, State Education Ministry in China (no. NCET10-0893), and the Natural Science Foundation of China (no. 6153367).

\section{References}

[1] C. E. Williams, "A Comparison of Circular Error Probable Estimators for Small Samples," DTIC Document, 1997.

[2] J. Zhang and W. An, "Assessing circular error probable when the errors are elliptical normal," Journal of Statistical Computation \& Simulation, vol. 82, no. 4, pp. 565-586, 2012.

[3] Y. Wang, G. Yang, D. Yan, Y. Wang, and X. Song, "Comprehensive assessment algorithm for calculating CEP of positioning accuracy," Measurement, vol. 47, no. 1, pp. 255-263, 2014.

[4] A. B. Chatfield, Fundamentals of High Accuracy Inertial Navigation, AIAA, Reston, Va, USA, 1997.

[5] G. Wang, X. Duan, and Z. Wang, "Optimal selection method of aim points based on hitting vital index of area target," Acta Aeronautica et Astronautica Sinica, vol. 29, no. 5, pp. 1258-1263, 2008.

[6] W. Gang, X. Duan, and Z. Wang, "Estimation method of firing accuracy for area targets based on Monte Carlo integral method," Systems Engineering and Electronics, vol. 7, article 36, 2009 (Chinese).

[7] J. C. Spall and J. L. Maryak, "A feasible Bayesian estimator of quantiles for projectile accuracy from non-iid data," Journal of the American Statistical Association, vol. 87, no. 419, pp. 676-681, 1992.

[8] T. Shi-Xue, "Introduction to the scene matching missile guidance technologies," in Proceedings of the Conference for 30th Anniversary of CSAA Establishment, 1994.

[9] B. Efron, "Bootstrap methods: another look at the jackknife," The Annals of Statistics, vol. 7, no. 1, pp. 1-26, 1979.

[10] J. MacKinnon, "Bootstrap hypothesis testing," Working Papers, vol. 74, no. 4, pp. 183-213, 2007. 


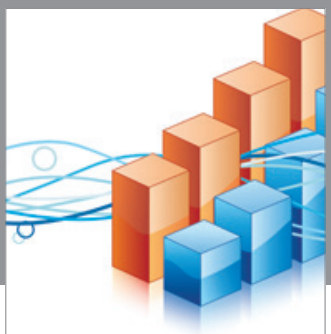

Advances in

Operations Research

vatem alat4

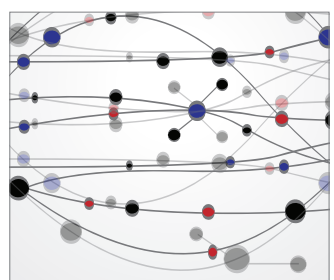

\section{The Scientific} World Journal
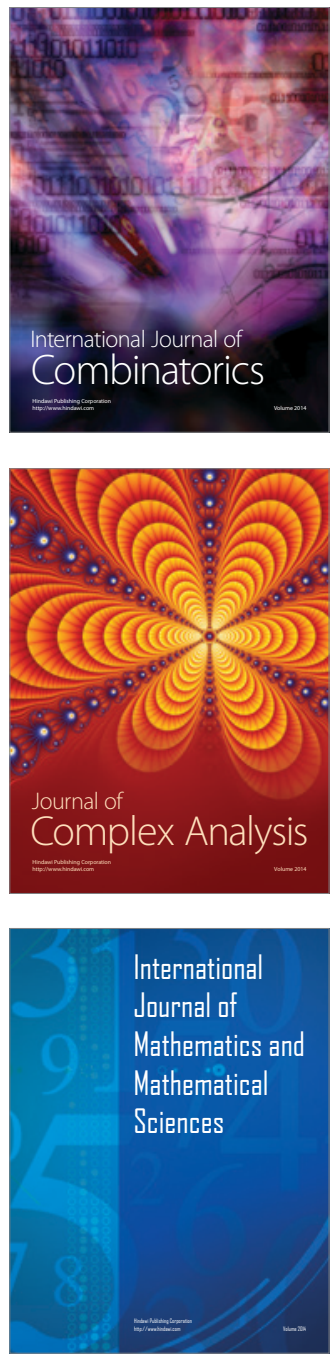
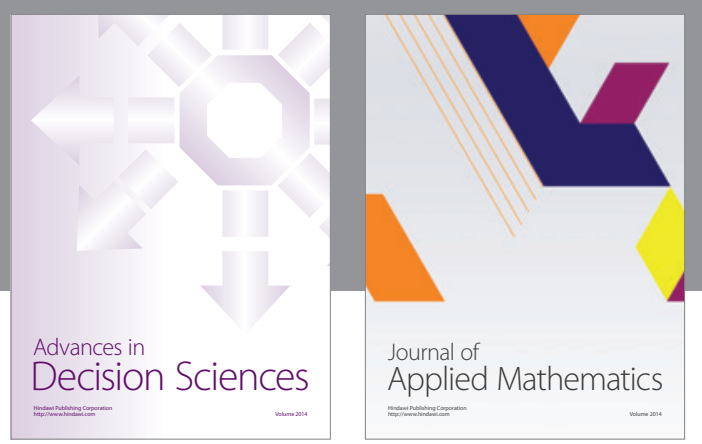

Algebra

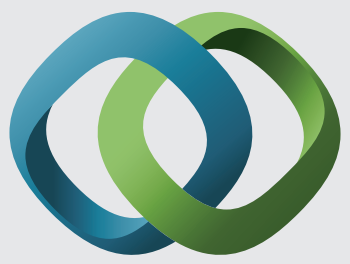

\section{Hindawi}

Submit your manuscripts at

https://www.hindawi.com
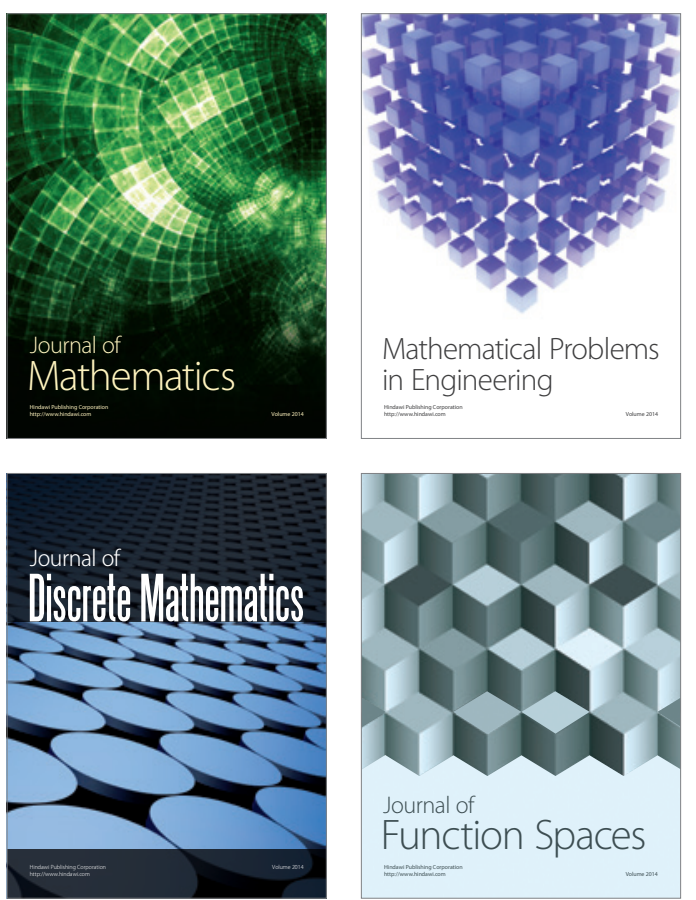

Mathematical Problems in Engineering
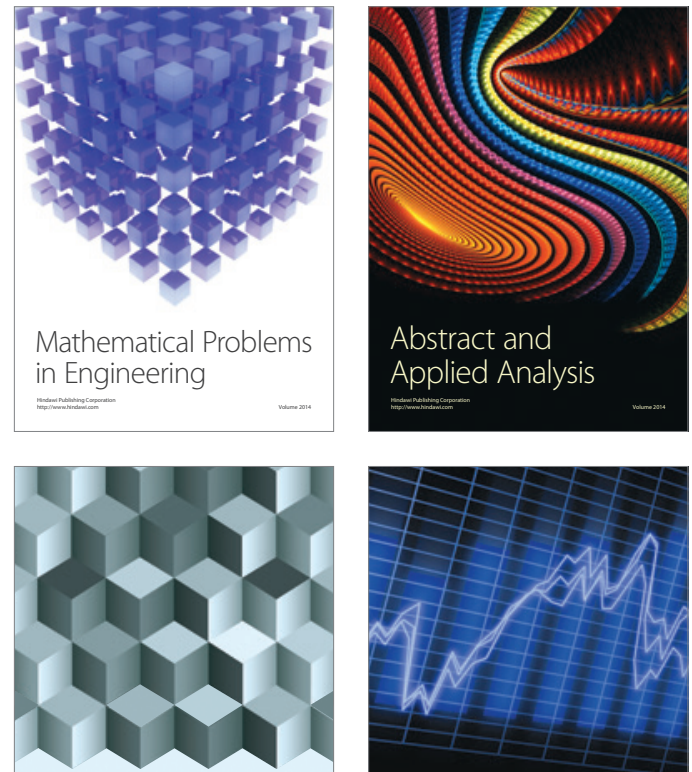

Journal of

Function Spaces

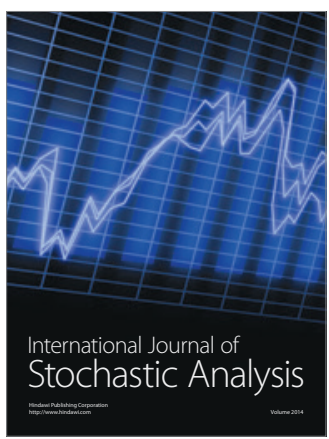

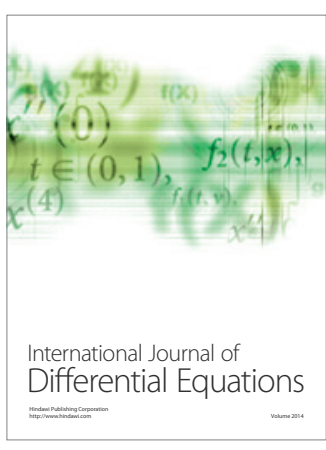
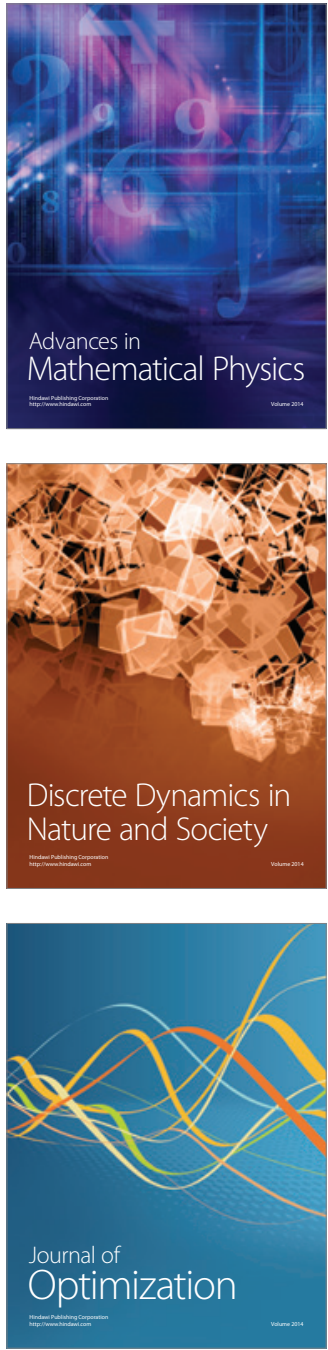